\title{
Non-charging Conditions of Insulating Film under Electron Beam Irradiation
}

\author{
Hideya Mizuno, ${ }^{\mathrm{a}}$ Kento Kubo, ${ }^{\mathrm{a}}$ Kentaro Kojima, ${ }^{\mathrm{b}}$ Masatoshi Kotera ${ }^{\mathrm{a}, \mathrm{b}, \uparrow}$ \\ a Major in Electrical and Electronic Engineering, Osaka Institute of Technology, 5-16-1, Omiya, Asahi-ku, Osaka, Japan \\ ${ }^{\mathrm{b}}$ Nanomaterials and Microdevices Research Center, Osaka Institute of Technology, 5-16-1, Omiya, Asahi-ku, Osaka, Japan \\ † Corresponding author: masatoshi.kotera@oit.ac.jp
}

Received: 10 December, 2019, Accepted 5 March, 2020, Published 21 March, 2020

It is known that insulating films charge up positively or negatively under electron beam irradiation. The potential distribution produced at the specimen surface can be measured by our homemade electrostatic force microscope installed in a scanning electron microscope. A global charging, found within several $\mathrm{mm}$ from the irradiation area is made by fogging electrons, which are generated by multiple backscattering events between the specimen and the objective lens plate. Since the most of the energy of fogging electrons is less than $5 \mathrm{eV}$, the global charging can be suppressed at the specimen by applying $-5 \mathrm{~V}$ to the specimen. On the other hand, the local charging at

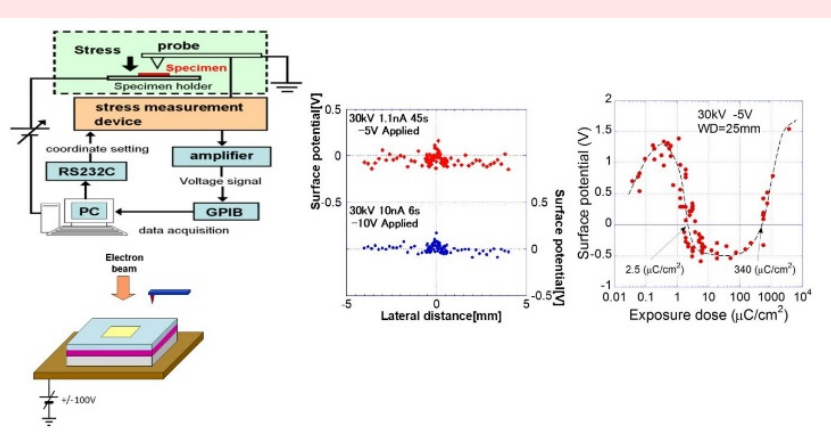
the irradiation point shows a positive charging in a small dose, but as the dose increases a negative charging is obtained. By increasing the dose further, a positive charging is found. We found two non-charging conditions of insulating film under electron beam irradiation.

Keywords Electron beam; Insulating film; Electrostatic force microscope; Fogging electrons; Non-charging condition

\section{INTRODUCTION}

In the large-scale integration (LSI) manufacturing process, it is important to manufacture a photomask with high accuracy. In order to realize a further high-speed and largescale semiconductor integrated circuit, it is necessary to further improve the electron beam (EB) drawing technologies. One of the troublesome phenomena in the process is charging of the photomask during EB exposure [1-9]. When the surface potential was measured with an electrostatic force microscope (EFM), it was confirmed that a potential distribution on the order of several $\mathrm{V}$ was present even at a position several $\mathrm{mm}$ away from the EB exposure point. It has been reported that the deviation of the exposure point from the designed position by this potential becomes a serious value of several tens of nanometers [10].

The resist charging phenomenon in the photomask manufacturing technology is a serious problem that must be solved in the future, and the purpose of this study is to search for the non-charging condition of the resist during electron beam exposure.

\section{EXPERIMENT}

There are only a few reports that directly measured the surface potential distribution, and the results with a spatial resolution of several millimeters are published around 1998 by a group led by Pease of Stanford University [11]. Figure 1 shows the schematic diagram of our EFM system installed in our scanning electron microscope (SEM) specimen chamber. Using the null-balance method, the surface potential of a specimen was obtained by measuring the force applied to the cantilever [7]. We measured the surface potential of a 300-nm-thick electron resist (FEP171) on 70-nm-thick $\mathrm{Cr}$ on a bulk glass substrate with $30 \mathrm{~mm} \times 60 \mathrm{~mm}$.

Figure 2 shows an illustration of the configuration of EB irradiation and the subsequent measurement of the surface potential distribution by the EFM cantilever probe. EB irradiation was done at $30 \mathrm{kV}$ accelerating voltage by a raster scan, and the area was a rectangle of $100 \mu \mathrm{m} \times 160 \mu \mathrm{m}$. By 


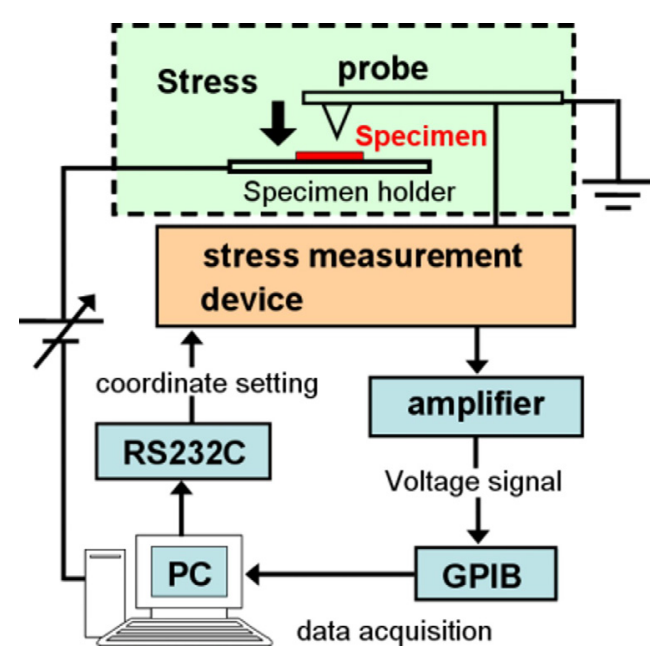

Figure 1: Schematic diagram of the present EFM system.

scanning the position of the probe, the spatial potential distribution was obtained.

During the EB irradiation, the EFM probe was kept about $100 \mathrm{~mm}$ away from the irradiating area, and after the irradiation the probe came back to the central field and the SEM stage was scanned to measure the potential distribution along the line, typically from $-4000 \mu \mathrm{m}$ to $4000 \mu \mathrm{m}$. To avoid influences of residual charge and the contact voltage difference of the system, the surface potential, which was measured before the EB irradiation, was subtracted from the potential after EB irradiation in every measurement.

\section{SURFACE POTENTIAL WHEN BIAS APPLICATION}

The EB irradiation was done as the time duration was $60 \mathrm{~s}$, a working distance (WD), a distance between the objective lens and the sample, was $25 \mathrm{~mm}$, and an acceleration voltage was $30 \mathrm{kV}$. After the EB irradiation, the sample bias was returned to $0 \mathrm{~V}$ and the surface potential was measured by EFM. As shown in Figure 2, the surface potential distribution of the sample was measured by changing the bias applied to the sample during beam irradiation from $-100 \mathrm{~V}$ to

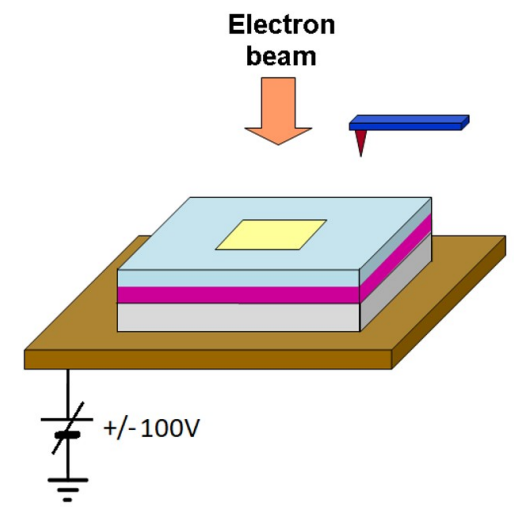

Figure 2: Configuration of EB irradiation and EFM measurement of the surface potential distribution.
$100 \mathrm{~V}$. The results, shown in Figure 3, are obtained with a spatial resolution of about $50 \mu \mathrm{m}$, which we know to be the finest [7]. This resolution was determined by the width of the cantilever. Paying attention to the large area outside the illumination range (maximum $\mathrm{mm}$ ), when a large positive bias such as $+100 \mathrm{~V}$ is applied, the surface will be at a fairly large negative potential. This global distribution is created by so-called fogging electrons (FGE) formed by multiple scattering of backscattered electrons between the sample and the objective lens bottom facing the sample. In contrast, when a negative bias such as $-100 \mathrm{~V}$ is applied, the surface is positively charged by emission from the surface of secondary electrons (SE) ionized by irradiation of high-speed FGE from the beam or the objective lens. On the other hand, it can be seen that the local potential directly under EB is less affected by the bias voltage.

\section{SEARCH FOR NON-CHARGING EXPOSURE CONDITIONS}

\section{A. Suppression of global charging}

First, a measured potential, which is shown in Figure 3 in the range of about $\pm 100 \mu \mathrm{m}$ was masked, and a potential distribution approximated by a Gaussian distribution was generated from outer distributions. The potential at the center of the distribution was extracted as the global charging potential. Figure 4 shows how these potential changes as the bias voltage changes. From this relationship, it was found that the surface potential becomes zero when the applied bias is about $-5 \mathrm{~V}$. This indicates that the energy of most FGE is less than $5 \mathrm{eV}$. The energy distribution of FGE has not been determined experimentally before, and there was

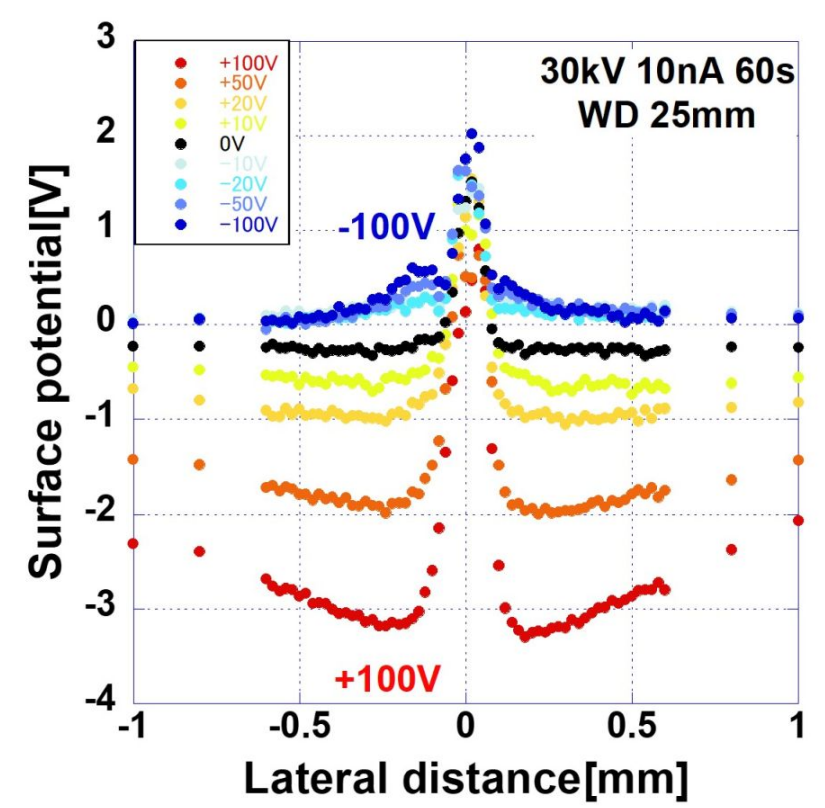

Figure 3: Potential distribution after EB exposure with bias applied. 


\section{eJSSNT}

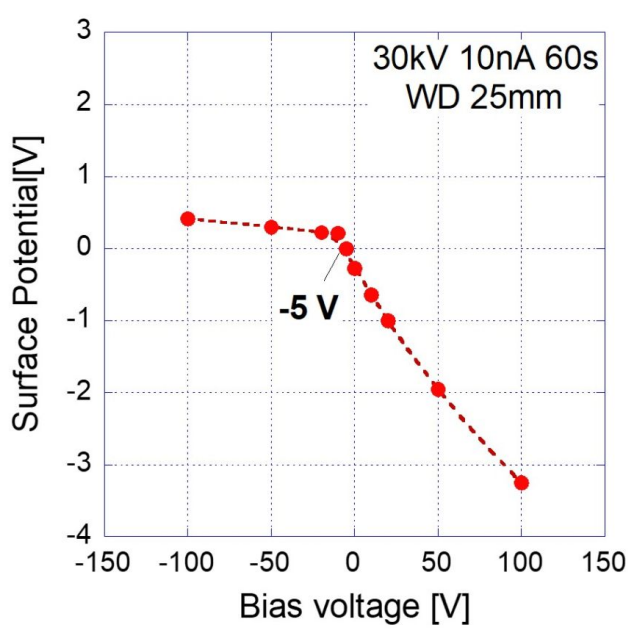

Figure 4: Bias voltage dependence of global charging potential.

no knowledge of how much potential should be applied to the specimen to suppress the global charging.

\section{B. Suppression of local charging}

Regarding the change in the local potential distribution just below the EB irradiation point, the change in the surface potential when the EB current during exposure was changed with an irradiation time of 60 seconds, the WD was $25 \mathrm{~mm}$, and the accelerating voltage of $30 \mathrm{kV}$ was obtained. As shown in Figure 5, the maximum local surface potential was found to increase with increasing current. Based on the relationship between the total amount of exposed charge and the surface potential, an uncharged state can be obtained when the amount of exposure was approximately $340 \mu \mathrm{C}$ $\mathrm{cm}^{-2}$.

From these results, the applied bias was set to about $-5 \mathrm{~V}$, and the results of the exposure amounts of $310 \mu \mathrm{C} \mathrm{cm}^{-2}(1.1$ $\mathrm{nA}, 45 \mathrm{~s})$ and $375 \mu \mathrm{C} \mathrm{\textrm {cm } ^ { - 2 }}(10 \mathrm{nA}, 6 \mathrm{~s})$ are compared (Figure 6). From these results, a non-charging condition was

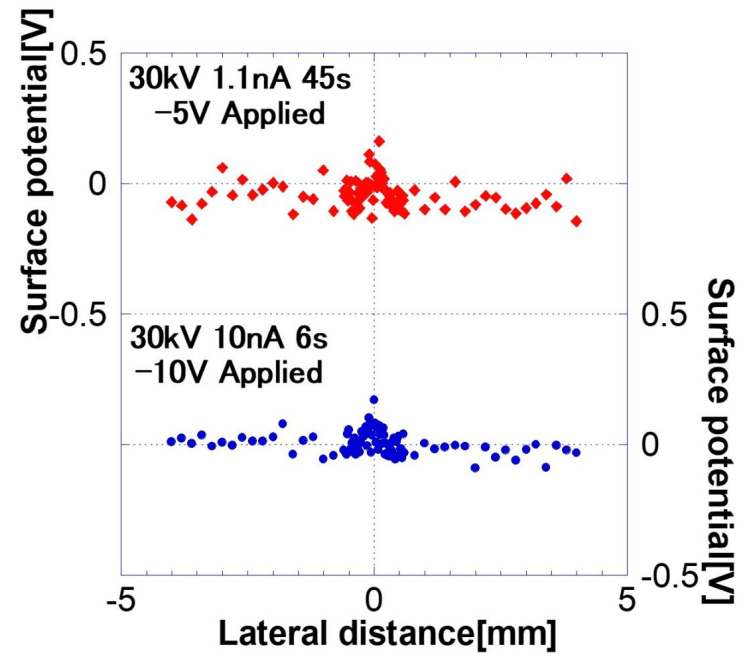

Figure 6: Non-charging condition.
ALC '19 Conference

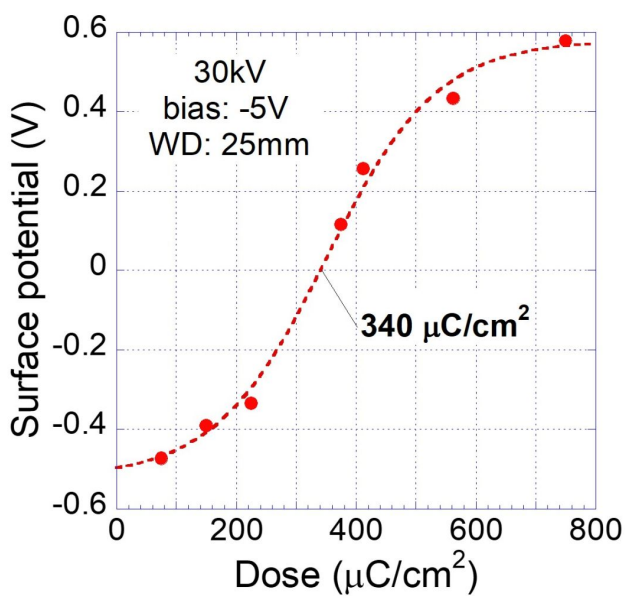

Figure 5: Dependence of local charging potential on total exposure.

realized that was within the potential of $0.1 \mathrm{~V}$ or less, which was the limit resolution of the EFM used for the surface potential measurement. In general, it is known that, as the irradiation dose increases, the sample accumulates electronic charge and becomes negatively charged. However, it is not previously known that, with further increases in exposure, the negative potential disappears and becomes positively charged instead.

\section{Surface potential over a wide range of doses}

Nakayamada et al. claimed that the surface potential could be zero at much lower doses [12]. Thus, we extended the exposure doses to a much lower value than that shown in Figure 5, we observed variations in surface potential, as shown in Figure 7 . This result certainly reproduces by the claim by Nakayamada et al. Low beam currents and/or short irradiation times were selected to achieve low doses.

The following process can be considered for this potential

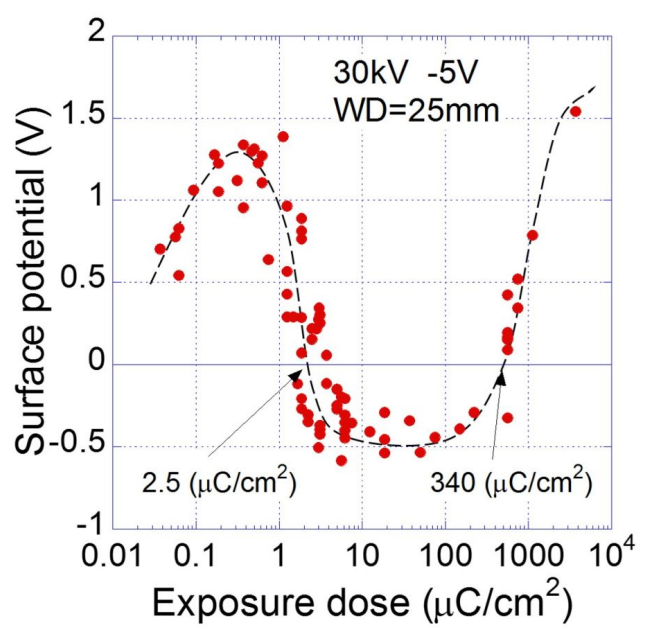

Figure 7: Changes in surface potential with changes in exposure dose. 
variation: At low exposure, the sample is charged positively by the emission of secondary electrons from the sample surface according to the SE yield. As the exposure dose increases, higher positive potentials can be expected due to increased SE emission. However, in practice, the positive potential on the specimen surface is saturated by the adsorption of electrons in vacuum after they are emitted. On the other hand, accumulation of negative charges due to the fact that electron charge reflected from the deeper bulk region accumulates in the sample and causes the negative potential. The surface potential is formed by the sum of these positive and negative charges. Since there is no process of migrating the accumulated electron charges, the sample shows a negative potential with an increase in the exposure dose. However, this negative potential disappears at a certain exposure dose as shown in Figure 5, and the sample surface becomes positively charged again at a higher dose. It is considered that this phenomenon occurs because an electron beam-induced conductive path is formed in the insulating thin film at such a large exposure dose. The result in Figure 7 indicates that there are two exposure doses of $2.5 \mu \mathrm{C} \mathrm{cm}{ }^{-2}$ and $337.5 \mu \mathrm{C} \mathrm{cm}^{-2}$ to give the specimen in the non-charging condition.

\section{SUMMARY}

In this study, we developed an EFM system that can measure the charged potential of EB irradiation and installed it in the SEM sample chamber. Using this system, we searched for exposure conditions to suppress the charging of the resist surface by EB irradiation for higher accuracy of EB lithography technology. It was found that FGE can be suppressed by applying a negative bias of $-5 \mathrm{~V}$ to the sample, and charging just under the beam can be suppressed by setting the exposure dose to appropriate values in $2.5 \mu \mathrm{C}$ $\mathrm{cm}^{-2}$ and $337.5 \mu \mathrm{C} \mathrm{cm}^{-2}$ as the dose.

\section{Acknowledgments}

In conducting this research, we received useful advice from Dr. Nakayamada, Dr. Kamikubo, and Dr. Nomura of NuFlare Technology Co., Ltd. A part of this research was done with Grant-inAid for Scientific Research (C) 19K04519.

\section{Note}

This paper was presented at the 12th International Symposium on Atomic Level Characterizations for New Materials and Devic- es '19 (ALC '19), in conjunction with the 22nd International Conference on Secondary Ion Mass Spectrometry (SIMS-22), Miyako Messe, Kyoto, Japan, 20-25 October, 2019.

\section{References}

[1] K. D. Cummings and M. Kiersh, J. Vac. Sci. Technol. B 7, 1536 (1989).

[2] N. Saitou, T. Iwasaki, and F. Murai, Proc. SPIE 1465, 185 (1991).

[3] M. Ogasawara, N. Shimomura, J. Takamatsu, S. Yoshitake, K. Ooki, N. Nakayamada, H. Okabe, T. Tojo, and T. Takigawa, J. Vac. Sci. Technol. B 17, 2936 (1999).

[4] N. Shimomura, M. Ogasawara, J. Takamatsu, S. Yoshitake, K. Ooki, N. Nakayamada, F. Okabe, and T. Tojo, Proc. SPIE 3748 , 408 (1999).

[5] M. Kotera and T. Maekawa, Jpn. J. Appl. Phys. 48, 06FB05 (2009).

[6] N. Nakayamada, T. Kamikubo, H. Anze, and S. Tamamushi, Proc. SPIE 8081, 808109 (2011).

[7] J. Kon, Y. Kojima, Y. Takahashi, T. Maruyama, and S. Sugatani, Jpn. J. Appl. Phys. 51, 06FC04 (2012).

[8] S. Babin, S. Borisov, and E. Patyukova, J. Vac. Sci. Technol. B 31, 06F411 (2013).

[9] T. Nishino, T. Noda, and M. Kotera, 10th International Symposium on Atomic Level Characterizations for New Materials and Devices '15 (Matsue, 2015) 27p-P-52.

[10] J. Cazaux, J. Appl. Phys. 85, 1137 (1999).

[11] M. Bai, D. S. Pickard, C. Tanasa, M. A. McCord, C. N. Berglund, and R. F. W. Pease, Proc. SPIE 3546, 383 (1998).

[12] N. Nakayamada, H. Nomura, and T. Kamikubo, 31st International Microprocesses and Nanotechnology Conference (Sapporo, 2018) 15A-5-1.

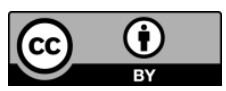

All articles published on e-J. Surf. Sci. Nanotechnol. are licensed under the Creative Commons Attribution 4.0 International (CC BY 4.0). You are free to copy and redistribute articles in any medium or format and also free to remix, transform, and build upon articles for any purpose (including a commercial use) as long as you give appropriate credit to the original source and provide a link to the Creative Commons (CC) license. If you modify the material, you must indicate changes in a proper way.

Published by The Japan Society of Vacuum and Surface Science 